\title{
GENERALIZED COMMUTATIVE RINGS
}

\author{
L. P. BELLUCE, I. N. HERSTEIN ${ }^{1)}$ and S. K. JAIN ${ }^{2)}$ \\ Dedicated to the memory of TaDasi Nakayama
}

Among his various interests in algebra Nakayama also took part in the various researches, published in the early and middle 1950's, which dealt with the commutativity of rings $[4,5]$. This paper, which studies a problem of a related sort, thus seems appropriate in a Journal honoring his memory.

We shall study a certain class of rings which satisfy a weak form of the commutative law and shall show that the structure of such rings can be determined.

Definition. A ring $R$ is said to be a generalized commutative ring (written as g.c. ring) if given $x, y \in R$ there exists positive integers $m(x, y), n(x, y)$ depending on $x$ and $y$ such that $(x y)^{n(x, y)}=(y x)^{m(x, y)}$.

We begin the study of g.c. rings with

THEOREM 1. Let $D$ be a division ring which is a g.c. ring; then $D$ is commutative.

Proof. Let $a, b \in D, a \neq 0, b \neq 0$; using $x=a, y=b a^{-1}$ in the definition of g.c. ring we know that there are positive integers $n$ and $m$ such that

$$
a b^{n} a^{-1}=\left(a b a^{-1}\right)^{n}=\left(a\left(b a^{-1}\right)\right)^{n}=\left(\left(b a^{-1}\right) a\right)^{m}=b^{m} .
$$

Therefore $a b^{n^{2}} a^{-1}=b^{m n}$ whence $a^{2} b^{n^{2}} a^{-2}=a b^{m n} a^{-1}=\left(a b^{n} a^{-1}\right)^{m}=b^{m^{2}}$. Continuing, we easily obtain

$$
a^{i} b^{n^{\iota}} a^{-i}=b^{m^{i}} \quad \text { for all } i>0 .
$$

Since $D$ is a g.c. ring there exist positive integers $k$ and $t$ such that

$$
b^{-n} a^{k} b^{n}=\left(b^{-n} a b^{n}\right)^{k}=a^{t} .
$$

Received November 16, 1964.

1) The work of Herstein was supported in part by a grant from the NSF, GP 2424 and one from the Army Research Office (AROD).

2) The work of Jain was supported in part by a grant from the NSF, GP 1447. 
Put $i=k$ in (2) and conjugate the result by $b^{n}$. We get

$$
a^{t} b^{n^{k}} a^{-t}=b^{m^{k}}
$$

Since, however, $a^{k} b^{n^{k}} a^{-k}=b^{m n^{k}}$ we see that $a^{t-k}$ commutes with $b^{n^{k}}$. If $k=t$ then (3) tells us that a positive power of $a$, namely $a^{k}$, commutes with a positive power of $b$, namely $b^{n}$. If $k \neq t$ the remark made above shows us that a positive power of $a$, namely $a^{|k-t|}$, commutes with the positive power $b^{n^{k}}$ of $b$. We have thus shown that for any $a, b \in D$ there exists positive integers $r(a$, $b), s(a, b)$ such that $a^{r(a, b)}$ commutes with $b^{s(a, b)}$. The proof of Theorem 1 will therefore be complete when we have established

Lemma 1. Let $D$ he a division ring in which given any $a, b$ there exist positive integers $r(a, b), s(a, b)$ such that $a^{r(a, b)}$ commutes with $b^{s(a, b)}$. Then $D$ is commutative.

Proof. If for every $x, y$ in $D, x$ commutes with some positive power of $y$, then by a result of Herstein [3] $D$ would be commutative.

Suppose then that we can find $a, b \in D$ such that $a$ commutes with no $b^{n}$ for $n>0$. Let $W=\left\{x \in D \mid x b^{m(x)}=b^{m(x)} x\right.$ for some $\left.m(x)>0\right\}$; Clearly $W$ is $a$ subdivision ring of $D$. Moreover, since $a \notin W, W \neq D$. By hypothesis, given $x \in D, x^{r(x, b)} \in W$, thus in Faith's terminology $D$ is radical over the proper subdivision ring $W$. A theorem of Faith [1] then tells us that $D$ is commutative.

Lemma 1 has some independent interest for from it one easily deduces that if $R$ is $a$ semi-simple ring in which $x^{r(x, y)}$ commutes with $y^{s(x, y)}$ for all $x, y$ in $R$ then $R$ is commutative. This answers a question raised by Faith [2].

Since subrings and homomorphic images of g.c. rings are g.c. rings and since the $n \times n$ matrices over a division ring (or any other ring with unit, for that matter) are not g.c. rings if $n>1$ we easily pass from the division ring case to the primitive case and thereby to the semi-simple case to obtain

THEOREM 2. A semi-simple g.c. ring is commutative.

CoRollary. If $R$ is a g.c. ring, $J(R)$ its Jacobson radical and $C(R)$ its commutator ideal then $C(R) \subset J(R)$.

We now are ready to prove the main result of this note

THEOREM 3. The commutator ideal of a g.c. ring is nil. 
Droof. Let $J(R)$ be the Jacobson radical of $R$ and $C(R)$ the commutator ideal of $R$. As we have just seen, $C(R) \subset J(R)$. Thus we may suppose that $J(R) \neq(0)$. By factoring out the maximal nil ideal of $R$ we may suppose that $R$ has no non-zero nil ideals. Our objective then becomes to prove that $R$ is commutative, that is, $C(R)=(0)$. Suppose $C(R) \neq(0)$.

We first claim that $J(R)$ can not be commutative, for if it were for $a, b \in$ $J(R)$ and $y \in R$ then since $a y \in J(R), a b y=b(a y)=(a y) b$, hence $a(b y-y b)=0$. Since $b y-y b$ is in $J(R) \cap\{x \in R \mid J(R) x=(0)\}$ which is a nilpotent ideal, we get that $b y-y b=0$ for all $b \in J(R), y \in R$, that is $J(R) \subset Z(R)$, the center of $R$. Given $a \in J(R), x, y \in R, a x \in J(R) \subset Z(R)$ hence $(a x) y=y(a x)=y a x=a y x$, leading to $a(x y-y x)=0$. Since $J(R) \subset Z(R)$ this immediately implies that $J(R) C(R)$ $=(0)$; together with $C(R) \subset J(R)$ we obtain $C(R)^{2}=(0)$. Since $R$ has no nonzero nilpotent ideals this latter forces $C(R)=(0)$, contrary to assumption.

We may therefore assume that $J(R)$ is not commutative. Since $R$ has no non-zero nil ideals then $J(R)$ as a ring in its own right also has no non-zero nil ideals. Since $J(R)$ has no nil ideals by the previously cited theorem of Herstein there are two elements $a, b \in J(R)$ such that $a$ commutes with no $b^{n}$ for $n>0$. Clearly then, $b$ can not be nilpotent.

Since $a \in J(R), 1-a$ is formally invertible ( $R$ need not have a unit element) and the mapping $x \rightarrow(1-a) x(1-a)^{-1}$ is an automorphism of $R$.

In the hypothesis that $R$ is a g.c. ring let $x=(1-a) b, y=b(1-a)^{-1}$. Thus there are positive integers $r, s$ such that

$$
\left((1-a) b^{2}(1-a)^{-1}\right)^{r}=\left(b(1-a)^{-1}(1-a) b\right)^{s}=b^{2 s},
$$

that is, there are positive integers $m, n>0(m=2 r, n=2 s)$ with

$$
(1-a) b^{m}=b^{n}(1-a) \text {. }
$$

Since $a$ commutes with no positive power of $b$ the integers $m, n$ in (1) satisfy $m \neq n$. We may suppose that $m>n$, otherwise we could carry out the argument on $a^{\prime}$ instead of $a$ where $(1-a)^{-1}=1-a^{\prime}$, that is, $a+a^{\prime}-a a^{\prime}=0$.

Since both $a b$ and $b a$ are in $J(R)$ the same reasoning yields

$$
\begin{aligned}
& (1-a b) b^{m}=b^{p}(1-a b) \\
& (1-b a) b^{m}=b^{q}(1-b a) .
\end{aligned}
$$

(We can suppose that the powers of $b$ on the left hand side of (1), (2) 
and (3) are the same for if $(1-a) b^{m_{1}}(1-a)^{-1}=b^{n_{1}},(1-a b) b^{m_{2}}(1-a b)^{-1}=b^{n_{2}}$, $(1-b a) b^{m_{3}}(1-b a)^{-1}=b^{n_{3}}$ then $m=m_{1} m_{2} m_{3}$ satisfies the conditions of (1), (2) and (3)).

Multiply (2) from the left by $b$ and (3) from the right by $b$; this yields

$$
\begin{aligned}
& (b-b a b) b^{m}=b^{p}(b-b a b) \\
& (b-b a b) b^{m}=b^{q}(b-b a b),
\end{aligned}
$$

hence $\left(b^{p}-b^{q}\right)(b-b a b)=0$.

If $p \neq q$ since $b$ is in the radical of $R$ (and so $\left(1-b^{k}\right) x=0$ forces $x=0$ when $k \geq 1)$ we get $b^{r+1}(1-a b)=0$ where $r=\min (p, q)$. Since $1-a b$ is invertible we are left with $b^{r+i}=0$, contrary to the fact that $b$ is not nilpotent. Therefore we conclude that $p=q$; (2) and (3) then become

$$
\begin{aligned}
& (1-a b) b^{m}=b^{p}(1-a b) \\
& (1-b a) b^{m}=b^{p}(1-b a) .
\end{aligned}
$$

Subtracting we get

$$
(a b-b a) b^{m}=b^{p}(a b-b a) .
$$

We return to the interrelation of (1) and (2). Multiply (1) from the right by $b$ and subtract from (2). We get

$$
b^{m+1}-b^{m}=b^{n+1}-b^{p}+\left(b^{n}-b^{p}\right) a b .
$$

If $b^{n}=b^{p}$ since $b \in J(R)$ we get $b^{k}=0$, where $k=\min (n, p)$, if $n \neq p$ contrary to $b$ not nilpotent, hence $b^{n}=b^{p}$ forces $n=p$. But then (5) reduces to $b^{m+1}-$ $b^{m}=b^{n+1}-b^{n}$. Since $m>n, b^{n}\left(1-b-b^{m-n}+b^{m+1-n}\right)=0$; however $b+b^{m-n}-$ $b^{m+1-n}$ being in $J(R), 1-b-b^{m-n}+b^{m+1-n}$ is invertible. The net result of this would be $b^{n}=0$, a contradiction. We thus know that $b^{n} \neq b^{p}$.

We commute (5) with $b$ to get $\left(b^{n}-b^{p}\right)(a b-b a) b=0$. Since $n \neq p$ and $b \subset J(R)$ this yields

$$
b^{t}(a b-b a) b=0 \quad \text { where } t=\min (n, p) .
$$

From (4) we know that

$$
(a b-b a) b^{k m}=b^{k p}(a b-b a) \quad \text { for any } k>0
$$

so, multiplying (6) from the left by $b^{k p-t}$ where $k p<t$ we get $(a b-b a) b^{k m+1}=0$, so certainly $(a b-b a) b^{(k+1) m}=0$. Since $0=(a b-b a) b^{(k+1) m}=b^{(k+1) p}(a b-b a)$, if 
$w=\max ((k+1) n,(k+1) p)$ then $(a b-b a) b^{w}=b^{w}(a b-b a)=0 . \quad$ A simple induction on $i$ reveals that

$$
0=\left(a b^{i}-b^{i} a\right) b^{w}=b^{w}\left(a b^{i}-b^{i} a\right) \quad \text { for all } i>0 .
$$

Put $i=w$; we then have $\left(a b^{w}-b^{w} a\right) b^{w}=0$ and $b^{w}\left(a b^{w}-b^{w} a\right)=0$. The net result of these is that $a b^{2 w}=b^{w} a b^{w}=b^{2 w} a$, that is, $a$ commutes with a positive power of $b$. This we know is contrary to assumption. The theorem is thereby proved.

\section{BIBLIOGRAPHY}

[1] C. Faith, Algebraic division ring extensions, Proc. A. M.S. vol. 11 (1960), pp. 43-53.

]2] C. Faith, Radical extensions of rings, Proc. A.M.S. vol. 12 (1961), pp. 274-283.

[3] I. N. Herstein, Two remarks on the commutativity of rings, Canadian J. Math. vol. 5 (1953), pp. 242-244.

[4] T. Nakayama, Über die Kommutativität gewisser Ringe, Abh. Math. Seminar Hamburg, vol. 20 (1955), pp. 20-27.

[5] T. Nakayama, A remark on the commutativity of algebraic rings, Nagoya Math. Jour. vol. 14 (1959), pp. 39-44.

University of California, Riversides

University of Chicago

University of California, Riversides 